\title{
ERBB4 promotes the proliferation of gastric cancer cells via the PI3K/Akt signaling pathway
}

\author{
$\mathrm{JI} \mathrm{XU}^{1-3^{*}}$, LIJIE GONG ${ }^{1,3,5^{*}}$, ZHENYUAN QIAN ${ }^{1-3^{*}}$, GUANGYUAN SONG ${ }^{6}$ and JINMING LIU ${ }^{2,4}$ \\ ${ }^{1}$ Department of Gastrointestinal and Pancreatic Surgery, Zhejiang Provincial People's Hospital; ${ }^{2}$ People's Hospital of \\ Hangzhou Medical College; ${ }^{3}$ Key Laboratory of Gastroenterology of Zhejiang Province; ${ }^{4}$ Department of Hepatobiliary and \\ Pancreatic Surgery, Zhejiang Provincial People's Hospital, Hangzhou, Zhejiang 310014 ; ${ }^{5}$ Zhejiang Chinese Medical University, \\ Hangzhou, Zhejiang 310053; ${ }^{6}$ Division of Hepatobiliary and Pancreatic Surgery, Department of Surgery, \\ First Affiliated Hospital, School of Medicine, Zhejiang University, Hangzhou, Zhejiang 310006, P.R. China
}

Received October 21, 2017; Accepted March 20, 2018

DOI: $10.3892 /$ or.2018.6343

\begin{abstract}
ERBB4 is one of the members of the epidermal growth factor receptor (EGFR) family. ERBB4 is a large transmembrane glycoprotein and has tyrosine kinase activity. Once combined with epidermal growth factor (EGF), ERBB4 can activate the related genes in the nucleus, thus promoting cell division and proliferation. In the present study, we investigated the effect of ERBB4 in the proliferation of gastric cancer cells. We found that high ERBB4 levels were closely related to the poor prognosis of gastric cancer patients. Furthermore, ERBB4 was highly expressed in gastric cancer cell lines when compared to the normal stomach cell line, GES. Clinical samples provided the same results. Two gastric cancer cell lines, SGC-7901 and MNK-45 were used to study the underlying mechanism of ERBB4 in the promotion of cell proliferation in gastric cancer cells both in vitro and in vivo. It was observed that after the expression of ERBB4 was suppressed, the proliferation of gastric cancer cells was markedly inhibited both in vitro and in vivo. Moreover, treatment with lentiviral vector siRNA-ERBB4 (Lv-siRNA-ERBB4) or the ERBB4 inhibitor AST-1306, markedly inhibited gastric cancer cell proliferation. Further experiments revealed that inhibition of the expression of ERBB4 could inhibit the activation of the PI3K/Akt signaling pathway. In addition, the use of the PI3K/Akt signaling pathway inhibitor LY294002 demonstrated the aforementioned results. Therefore, we believe that ERBB4 regulates cell proliferation mainly through the PI3K signaling pathway. Finally, nude mice xenografted with gastric cancer cells with low expression of ERBB4 exhibited smaller tumors and longer survival than those engrafted with control gastric cancer cells. These data
\end{abstract}

Correspondence to: Dr Jinming Liu, Department of Hepatobiliary and Pancreatic Surgery, Zhejiang Provincial People's Hospital, 168 Shangtang Road, Xiacheng, Hangzhou, Zhejiang 310014, P.R. China E-mail: ljmhsq@163.com

*Contributed equally

Key words: ERBB4, proliferation, gastric cancer, PI3K-Akt pathway indicated that ERBB4 promoted cell proliferation and is thus a potential therapeutic target for the treatment of gastric cancer.

\section{Introduction}

Gastric cancer has a high incidence and mortality in China (1). Because the symptoms of early gastric cancer are not obvious, most cases of tumor invasion and metastasis are diagnosed only when patients are hospitalized (2). Low chemotherapy sensitivity results in poor survival rates among gastric cancer patients $(2,3)$. Therefore, finding effective targets to inhibit the growth and metastasis of gastric cancer is particularly important. ERBB4 is a large transmembrane glycoprotein, and has tyrosine kinase activity (4). Once combined with epidermal growth factor (EGF), ERBB4 can activate the related genes in the nucleus, thus promoting cell division and proliferation $(5,6)$. The expression of ERBB4 was revealed to be increased in gastric, breast, and bladder cancer as well as head and neck squamous cell carcinoma (7-10). In addition, ERBB4 has been revealed to contribute to and play an important role in tumor cell survival, proliferation, and motility $(11,12)$. ERBB4 has been demonstrated to be overexpressed or with aberrant expression in a variety of solid tumors especially in gastric cancer (13-16). Therefore, ERBB4 may become a very important drug target for the therapy of gastric cancer. Unfortunately, more research attention has been paid to ERBB2 overlooking ERBB4. In the present study, we investigated the effect of ERBB4 in the proliferation of gastric cancer cells. We found that high ERBB4 levels were closely related to the poor prognosis of gastric cancer patients. ERBB4 was highly expressed in gastric cancer cell lines when compared to the normal stomach cell line, GES. Clinical samples provided the same results. We also found that ERBB4 regulated cell proliferation mainly through the PI3K/Akt signaling pathway. Thus, ERBB4 may become a potential therapeutic target for the treatment of gastric cancer.

\section{Materials and methods}

Patients and tissue specimens. Gastric cancer tumor tissues with matched adjacent non-tumor tissues were obtained from 27 patients that underwent surgical treatment at Zhejiang 
Provincial People's Hospital (Hangzhou, China). All patients provided a signed informed consent. None of them had a history of chemotherapy or radiotherapy before sampling, and the diagnosis of gastric cancer was pathologically confirmed. This study was approved by the Institutional Ethics Committee of Zhejiang Provincial People's Hospital.

Reagents and antibodies. RPMI-1640 was acquired from Thermo Fisher Scientific, Inc. (Waltham, MA, USA). Fetal bovine serum (FBS) was purchased from Gibco (Rockville, MD, USA). Inhibitors AST-1306 and LY294002 were obtained from Selleck Chemicals (Houston, TX, USA). A BCA Protein Assay kit was purchased from Beyotime Institute of Biotechnology (Shanghai, China). Antibodies against $\beta$-actin (cat. no. 4970S), ERBB4 (cat. no. 4795S), PI3K (cat. no. 4249S), Akt (cat. no. 9272S), and p70S6K (cat. no. 2708S) and secondary antibodies (cat. no. 7054S) were purchased from Cell Signaling Technology, Inc. (Danvers, MA, USA). All antibody dilution ratio was 1:1,000. MTT was purchased from Beyotime Institute of Biotechnology. Other chemicals were obtained from commercial sources. An IHC staining kit was purchased from Thermo Fisher Scientific, Inc.

Cell culture and transfection. Gastric cancer cell lines MNK-45 and SGC-7901 were acquired from the Cell Bank of the Chinese Academy of Sciences (Shanghai Institute of Cell Biology, Shanghai, China). MNK-45 and SGC-7901 cells were cultured in RPMI-1640 containing 10\% FBS and maintained at $37^{\circ} \mathrm{C}$ in an atmosphere of $5 \% \mathrm{CO}_{2}$ in a humidified incubator. MNK-45 and SGC-7901 cells $\left(1.5 \times 10^{5}\right)$ were seeded in 6-well plates, and incubated for $12 \mathrm{~h}$, then transfected with a lentiviral vector encoding small interfering RNA targeting ERBB4 [lentiviral vector siRNA-ERBB4 (Lv-siRNA-ERBB4)] and negative control lentiviral vector ( $\mathrm{Lv}-\mathrm{NC})$. Lv-siRNA-ERBB4 and Lv-NC were synthesized by Shanghai GeneChem, Co., Ltd. (Shanghai, China). ViralPlus Transduction Enhancer and Polybrene were used for lentiviral vector transfection.

Western blot analysis. Cells or tissues were lysed using RIPA buffer containing $1 \mathrm{mM}$ PMSF protease inhibitor mixture. The total protein concentration was assessed using the BCA assay and was equalized with the extraction reagent. Protein (10 $\mu \mathrm{g} /$ lane) samples were separated by $10 \%$ SDS-PAGE, and electrotransferred onto PVDF nitrocellulose membranes The membranes were blocked with $5 \%$ skim milk for $2 \mathrm{~h}$ at $37^{\circ} \mathrm{C}$. Then, the membranes were incubated with primary antibodies against $\beta$-actin (cat. no. 4970S), ERBB4 (cat. no. 4795S), PI3K (cat. no. 4249S) Akt (cat. no. 9272S) and secondary antibody anti-rabbit IgG (cat. no. 7054S; all from Cell Signaling Technology, Danvers, MA, USA) and diluted 1:1,000. Subsequently, a Gel imaging analysis system and Pierce Western Blot Signal Enhancer (cat. no. 21050; Thermo Fisher Scientific, Inc.) were used to detect the protein bands.

Cell proliferation assay. The cell proliferation of MNK-45 and SGC-7901 cells was analyzed using flow cytometry. Briefly, the cells were plated at a density of 1,000-10,000 cells/well in a 96-well plate, and incubated at $37^{\circ} \mathrm{C}$ for $12,24,36$, 48 and $60 \mathrm{~h}$. At 12, 24, 48 or $60 \mathrm{~h}$, the cells were incubated with RPMI-1640 medium containing $0.5 \mathrm{mg} / \mathrm{ml} \mathrm{MTT}$ at $37^{\circ} \mathrm{C}$ for $4 \mathrm{~h}$. Formazan crystals were dissolved with $150 \mu \mathrm{l}$ DMSO. The absorbance of each well, including blanks, was measured at $490 \mathrm{~nm}$ using an automatic microplate reader subsequent to 10 min of oscillation. The formula of the cell growth inhibition rate was as follows: Inhibition rate $=(1-$ absorbance of the COE group/absorbance of the blank control group) $\mathrm{x} 100 \%$.

In vivo imaging technology. In order to further examine the effect of ERBB4 on the growth of gastric cancer cells in vivo, the animal imaging technique was used to perform the assay in vivo. Four-week-old nude mice were purchased from the Laboratory Animal Center of Zhejiang University. All animal experiments were performed in accordance with the guidelines of the National Institutes of Health Guide for the Care and Use of Laboratory Animals. Human gastric cancer cell lines MNK-45 were transfected with Lv-siRNA-ERBB4-GFP and Lv-NC-GFP and then transplanted subcutaneously in nude mice. Tumor cells labeled with green fluorescence were used for in vivo fluorescence imaging after 7 days. The initial inoculum concentration of MNK-45 was increased to $1 \times 10^{7}$ cells $/ \mathrm{ml}$. Tumors were formed $\sim 7$ days after the injection. Tumors sizes were measured every 7 days using an in vivo imaging system (PerkinElmer, Inc., Waltham, MA, USA). The fluorescence signal reflecting the tumor sizes of the mice were collected and analyzed using the in vivo imaging system. This assay was approved by the Institutional Ethics Committee of Zhejiang Provincial People's Hospital.

Immunohistochemistry. All specimens were fixed in neutral buffered formalin and embedded in paraffin. The specimens were cut into 5- $\mu \mathrm{m}$ sections, deparaffinized in xylene, and rehydrated in graded ethanol. After non-specific binding sites were blocked by exposing them to $10 \%$ normal goat serum in PBS for $20 \mathrm{~min}$, the sections were incubated overnight at $4^{\circ} \mathrm{C}$ using a series of antibodies (ERBB4, 1:200; PI3K, 1:200; Akt, 1:200). Following this incubation, the slides were rinsed with PBS and incubated with biotinylated $\mathrm{IgG}$ for $20 \mathrm{~min}$ at $37^{\circ} \mathrm{C}$. Finally, the sections were slightly counterstained with hematoxylin for $30 \mathrm{sec}$ before coverslip mounting.

Statistical analysis. The data processing was carried out using SPSS 19.0 statistical software. One-way analysis of variance (ANOVA) with Dunnett's test was used to determine statistically significant differences. All data were expressed as the mean \pm standard deviation $(\mathrm{x} \pm \mathrm{s}) . \mathrm{P}<0.05$ and $\mathrm{P}<0.01$ indicated differences that were statistically significant. All experiments were repeated at least three times.

\section{Results}

ERBB4 is downregulated in gastric cancer. We analyzed 378 gastric cancer samples obtained from The Cancer Genome Atlas (TCGA) datasets to identify critical ERBB4 involved in gastric cancer. The analysis demonstrated that high ERBB4 levels in TCGA gastric cancer data were closely related to the poor survival rate of gastric cancer patients suggesting that ERBB4 was a prognostic indicator of gastric cancer (Fig. 1A). Quantification of the western blot analysis was performed. In addition, western blot analysis revealed that ERBB4 was significantly overexpressed in MNK-45 and SGC-7901 gastric cancer cell lines compared to immortalized stomach GES cells (Fig. 1B). 

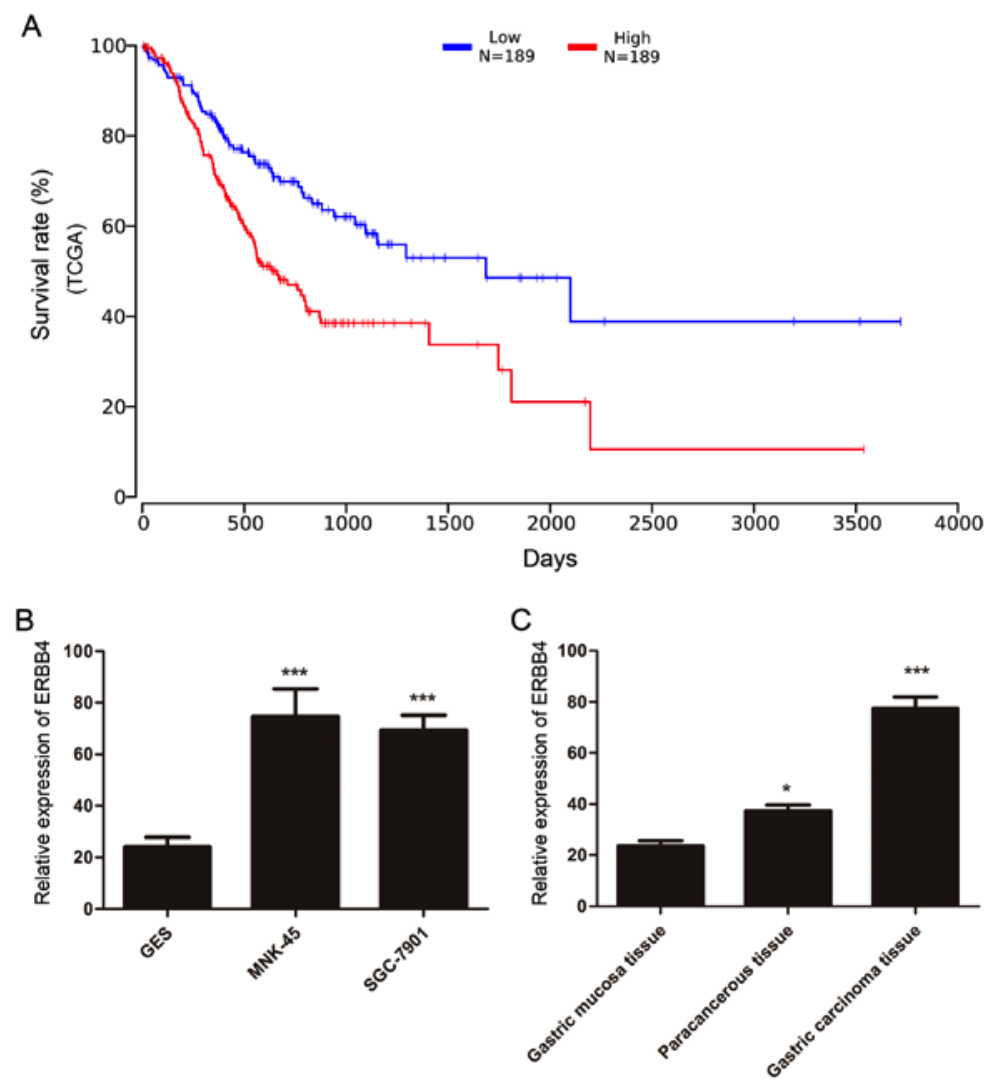

Figure 1. (A) The plot (data from the TCGA database) shows the survival rate (\%) of gastric cancer patients with high (red) and low (blue) expression of ERBB4. Low expression of ERBB4 was associated with long term survival. (B) The expression levels of ERBB4 in GES, MNK-45 and SGC-7901 cells. (C) ERBB4 expression in normal stomach, paracancerous and gastric cancer tissues. ${ }^{*} \mathrm{P}<0.05,{ }^{* * * *} \mathrm{P}<0.001$. TCGA, The Cancer Genome Atlas.

Furthermore, western blot analysis also revealed that ERBB4 was markedly upregulated in tumor tissues compared to normal stomach tissues and paracancerous tissue (Fig. 1C).

A lentivirus interferes with the expression of ERBB4. To further study the function of ERBB4 in gastric cancer, lentiviral vectors encoding siRNAs targeting ERBB4 (GenBank accession number NC_000002.12) were constructed by Shanghai GeneChem, Co., Ltd. Three different siRNAs targeting ERBB4 (Lv-siRNA-ERBB4) were designed to ensure the interference effect. The three Lv-siRNA-ERBB4 vectors were transfected into MNK-45 and SGC-7901 cells with ViralPlus Transduction Enhancerand Polybrene so that theirspecificity for ERBB4 disruption could be determined. The validated Lv-siRNA-ERBB4 was selected for the construction of the lentiviral vector (Fig. 2A-C). A non-silencing sequence was used as a negative control (Lv-NC). Western blot analysis revealed that ERBB4 was markedly silenced by Lv-siRNA-ERBB4 (Fig. 2D and E).

Effects of ERBB4 silencing on gastric cancer cell proliferation and related signaling pathways. The MTT assay revealed that after Lv-siRNA silenced ERBB4 expression, MNK-45 and SGC-7901 cell viability and cell proliferation was decreased. Compared with the control group, the cells after Lv-siRNA silenced ERBB4 expression exhibited a marked growth inhibition (Fig. 3A and B). Western blot analysis revealed that ERBB4 silencing, inhibited the downstream PI3K/Akt signaling. Furthermore, the same results were obtained with the ERBB4 inhibitor, AST-1306. In addition, the PI3K/Akt signaling inhibitor LY294002 also provided further evidence, constistent with the aforementioned results. (Fig. 3C-F).

Silencing of ERBB 4 expression suppresses tumor growth in vivo and prolongs the survival time. To confirm the effects of ERBB4 in vivo, we used a xenograft mouse model. The analysis demonstrated that Lv-NC cells formed significantly larger tumors than the Lv-siRNA-ERBB4 group of cells in the nude mice (Fig. 4A). In addition, the body weight of nude mice exhibited no difference (Fig. 4B). Furthermore, the tumor volume was significantly diminished in the Lv-siRNA-ERBB4 group compared to the Lv-NC group (Fig. 4C). Notably, silencing of ERBB4 expression resulted in a significantly enhanced survival time of the tumor-bearing nude mice (Fig. 4D).

Lv-siRNA and AST-1306 markedly inhibit the growth of transplanted tumors in nude mice. Lv-siRNA-ERBB4 significantly inhibited tumor growth in nude mice. The size of the transplanted tumors in nude mice treated with AST-1306 or transfected with the lentivirus was significantly lower than that in the Lv-NC group (Fig. 5).

AST-1306 regulates ERBB4-PI3K/Akt signaling pathways in vivo. To further ascertain the signal transduction pathway mediating the anti-proliferation effects in vivo, xenografts of nude mice were prepared as paraffin-embedded sections. The IHC analysis revealed that the inhibitor of ERBB4 (AST-1306) significantly downregulated the expression level of the proliferation-related proteins PI3K and Akt (Fig. 6). 
A
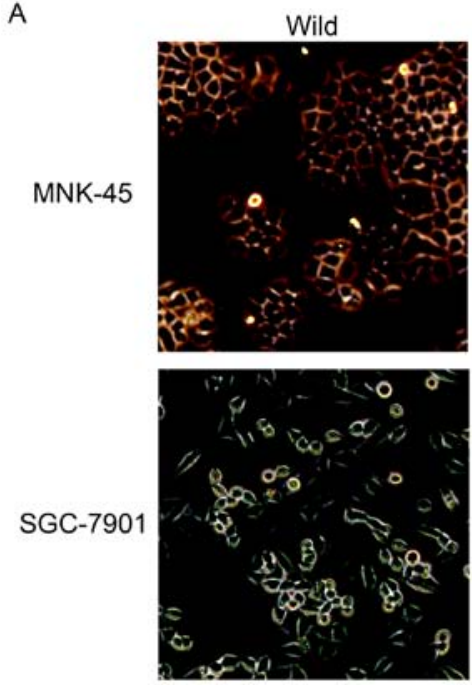

B

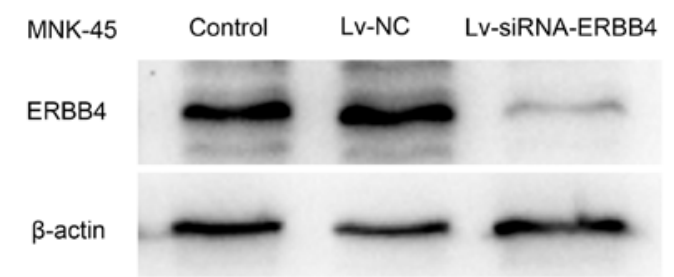

D

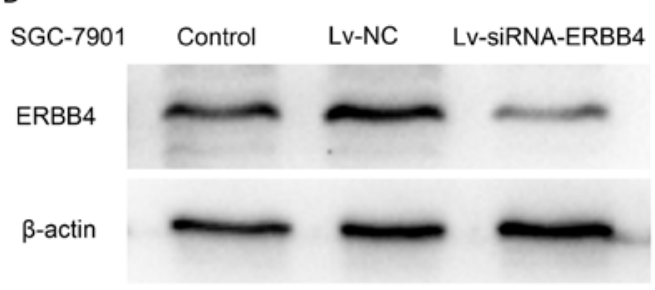

Lv-siRNA-ERBB4
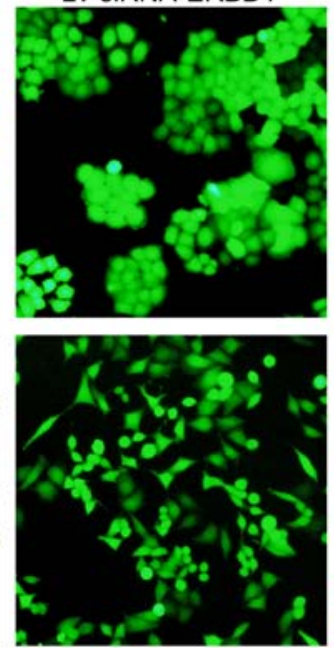
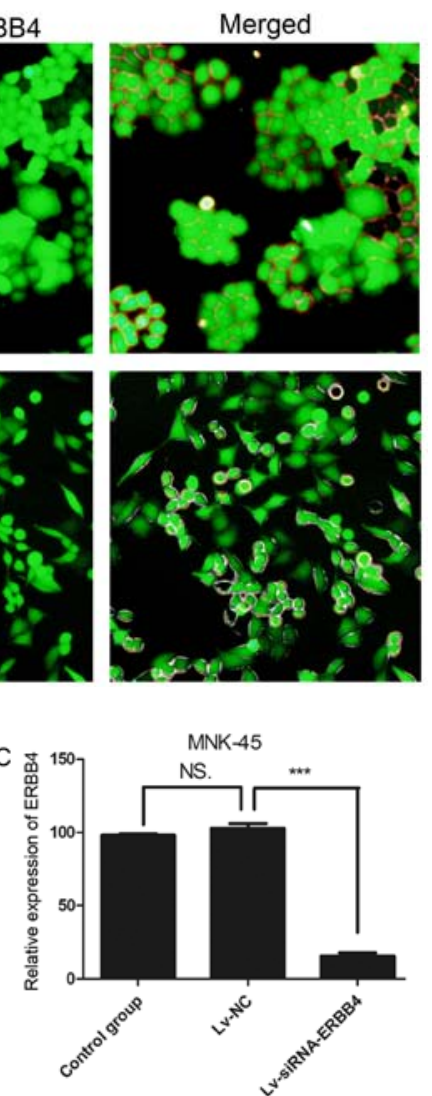

E

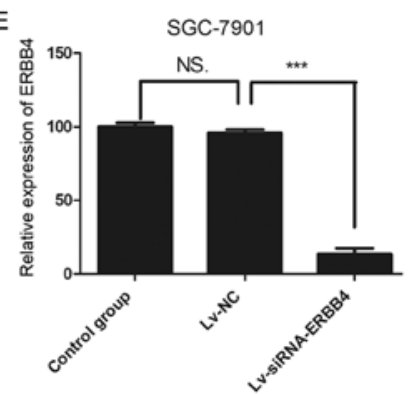

Figure 2. Lv-siRNA-ERBB4 silenced ERBB4 expression (x100). (A) Expression of green fluorescent protein before and after transfection with the lentivirus. (B-E) Lv-siRNA-ERBB4 markedly downregulated ERBB4 expression in MNK-45 and SGC-7901 cells. ${ }^{* * *} \mathrm{P}<0.001$. Lv-siRNA-ERBB4, lentiviral vector siRNA-ERBB4; Lv-NC, negative control lentiviral vector.

\section{Discussion}

Gastric cancer is one of the most common cancers with the highest morbidity and mortality in China (1). Early screening of gastric cancer is an important method for the treatment and prevention of gastric cancer (17-20). However, there is no reliable and specific molecular marker of gastric cancer. Therefore, it is important to find a specific marker of gastric cancer. ERBB4 belongs to the family of tyrosine kinase receptors and is overexpressed in many tumors especially in gastric cancer. In recent years, the targeted therapy of epidermal growth factor receptor (EGFR) family has achieved fruitful results in the treatment of gastric cancer (21-24). The EGFR family was determined to be highly expressed in gastric cancer, and the positive rate of EGFR (ERBB1/HER1) was 40-60\% (25), human epidermal growth factor receptor-2 (ERBB2/ HER-2) ranged from 4 to 53\% (26), HER-3/ERBB3 and HER-4/ERBB4 were 59 and $86 \%$, respectively (27). The positive expression rate of ERBB4 in gastric cancer was found to be the highest, but few studies have been performed on ERBB4 in gastric cancer. In the present study, we found that the high expression of ERBB4 was closely related to the poor prognosis of the patients. Inhibiting the expression of ERBB4 contributed to the prolongation of the survival time of gastric cancer patients. Through experiments, we found that ERBB4 was stably expressed in gastric cancer tissues and cells relative to normal gastric mucosa tissues and gastric mucosa cells. Therefore, we hypothesized that ERBB4 may play an important role in the malignant behavior of gastric cancer. It may become a target for predicting the prognosis and treatment for gastric cancer patients. To further investigate the role and mechanism of ERBB4 in gastric cancer, we used molecular cloning techniques and a lentivirus to inhibit the expression of ERBB4. The results revealed that the proliferation of gastric cancer cells was markedly inhibited by silencing the expression of ERBB4. Thus, ERBB4 can promote the proliferation of gastric 

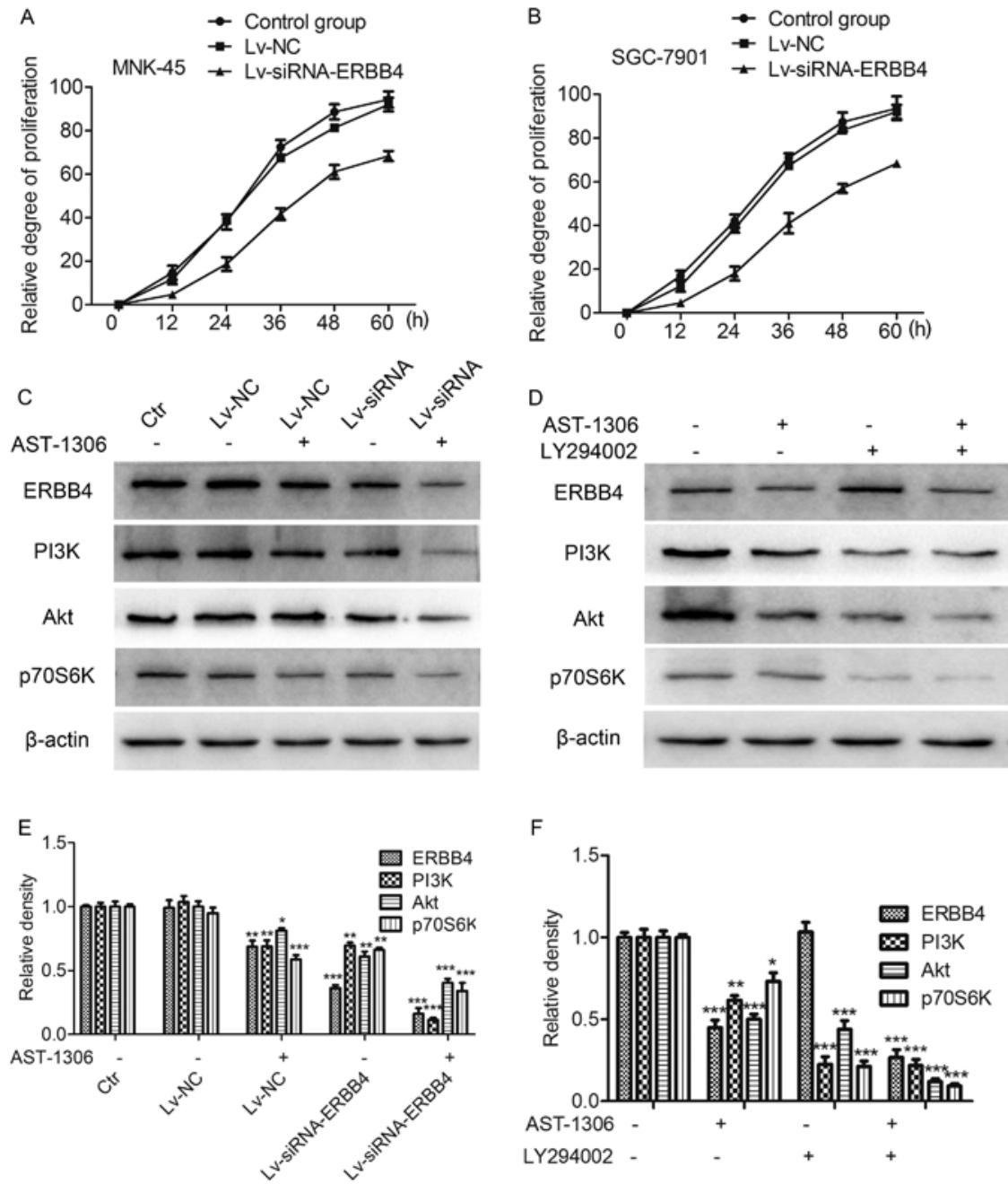

$\mathrm{F}$

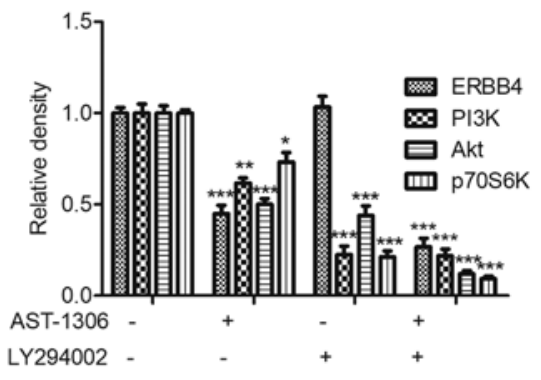

Figure 3. Inhibition of ERBB4 expression on the proliferation of gastric cancer cells (A) MNK-45 and (B) SGC-7901. (C-F) Silencing of ERBB4 expression, inhibited the downstream PI3K/Akt signaling. Two inhibitors upstream and downstream demonstrated the effects of ERBB4 on the PI3K/Akt signaling pathway. ${ }^{*} \mathrm{P}<0.05,{ }^{* *} \mathrm{P}<0.01,{ }^{* * *} \mathrm{P}<0.001$. Lv-NC, negative control lentiviral vector; Lv-siRNA-ERBB4, lentiviral vector siRNA-ERBB4.

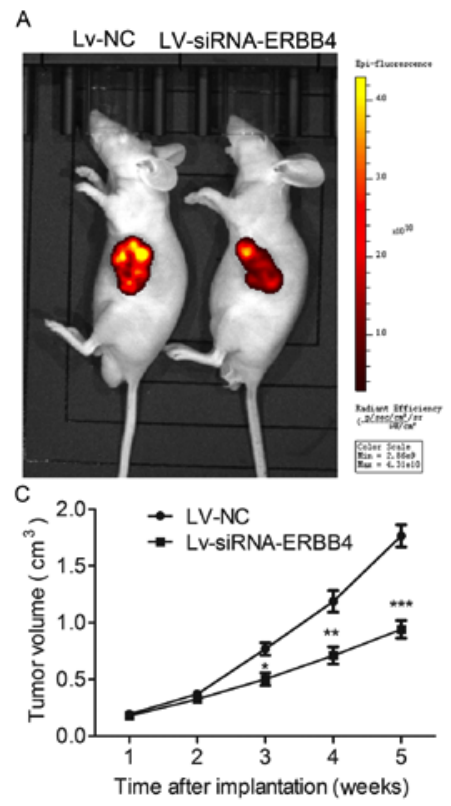

B
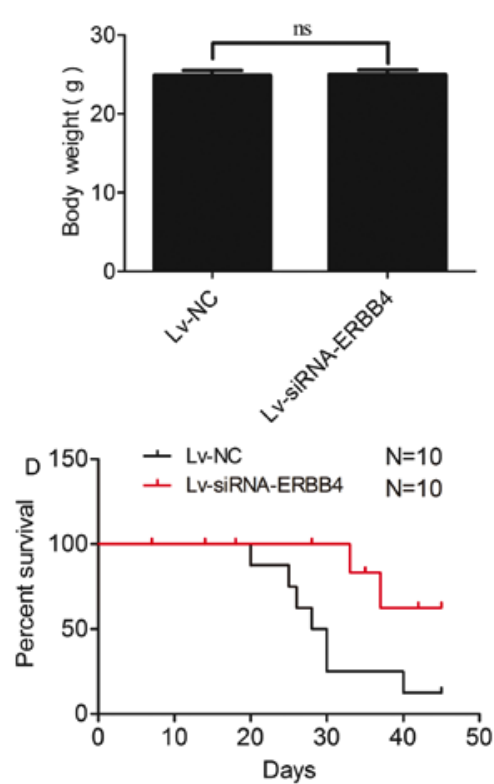

Figure 4. Xenograft mouse model reveals that silencing ERBB4 expression significantly inhibits tumor growth in vivo. (A) Live imaging of subcutaneous tumor growth at 21 days in the $\mathrm{Lv}-\mathrm{NC}$ and Lv-siRNA-ERBB4 groups in nude mice. (B) Body weight of nude mice. (C) Tumor volume curves. (D) Comparison of the survival curves of nude mice injected with gastric cancer cells that are either Lv-NC (black) or Lv-siRNA-ERBB4 (red). ${ }^{*} \mathrm{P}<0.05,{ }^{* *} \mathrm{P}<0.01,{ }^{* * * *} \mathrm{P}<0.001 . \mathrm{Lv}-\mathrm{NC}$, negative control lentiviral vector; Lv-siRNA-ERBB4, lentiviral vector siRNA-ERBB4. 

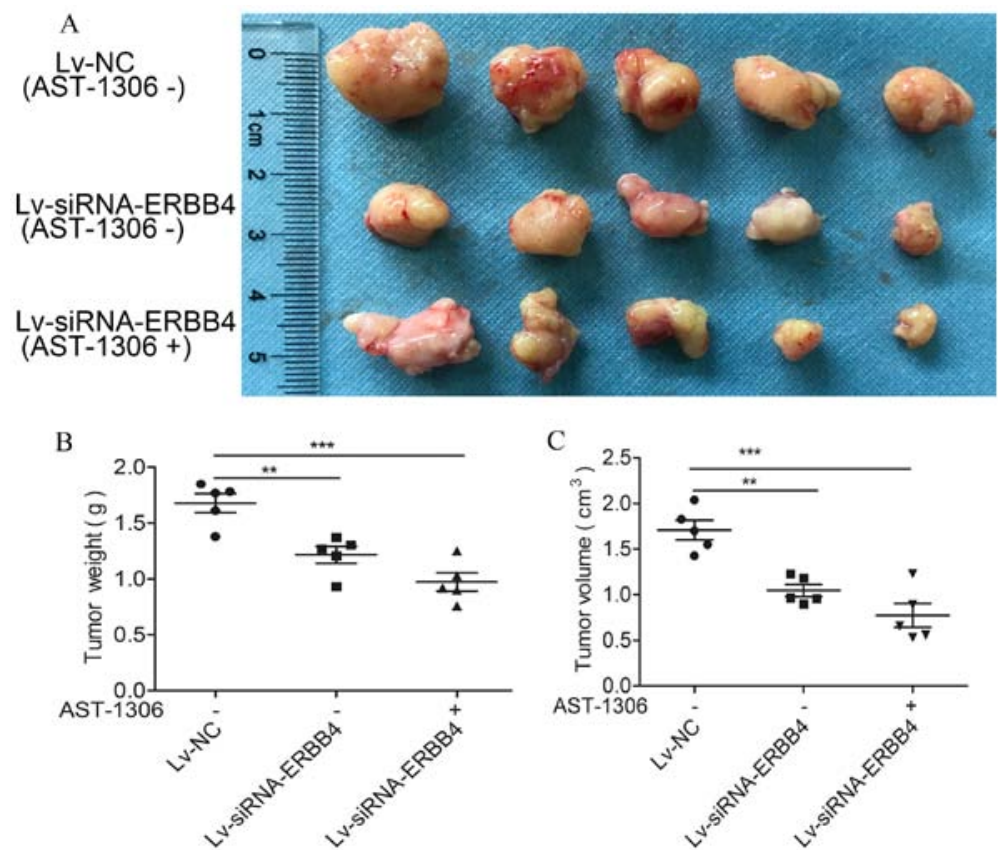

Figure 5. (A) Tumor size of different groups, including Lv-NC group, Lv-siRNA-ERBB4 group and combined use of the lentivirus and AST-1306 group. (B) Tumor weight and (C) volume of nude mice. Lv-NC, negative control lentiviral vector; Lv-siRNA-ERBB4, lentiviral vector siRNA-ERBB4.

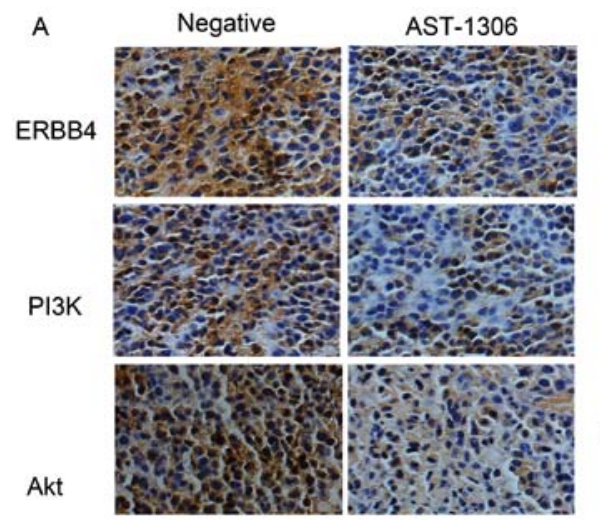

B

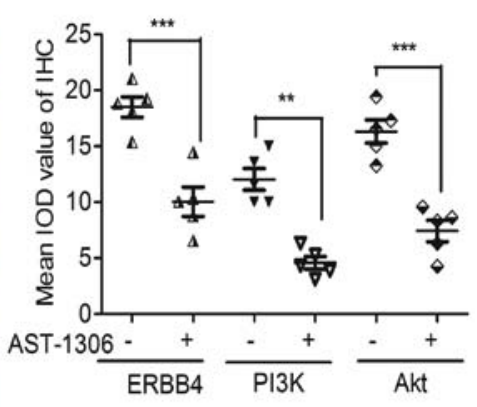

Figure 6. (A and B) AST-1306 downregulates the levels of the proliferation-related proteins ERBB4, PI $3 K$ and Akt. ${ }^{* * *} \mathrm{P}<0.01,{ }^{* * * *} \mathrm{P}<0.001$

cancer cells. We further detected the key proteins of the downstream signaling pathway of ERBB4 and found that inhibition of ERBB4 expression would directly result in inactivation of the downstream PI3K/Akt signaling pathway. In addition, the PI3K signaling pathway can directly regulate cell proliferation through its downstream proteins m-TOR and p70S6K (28-30). These results revealed that ERBB4 can promote the proliferation of gastric cancer cells through the PI3K/Akt signaling pathway. In addition, the ERBB4 inhibitor (AST-1306) and the PI3K inhibitor (LY-294002) experiments confirmed the aforementioned conclusion. Collectively, these results indicated that ERBB4 mainly promotes the proliferation of gastric cancer cells through the PI3K/Akt signaling pathway. In order to investigate the role and mechanism of ERBB4 on gastric cancer proliferation in vivo, xenograft models of gastric cancer and an in vivo imaging system (PerkinElmer, Inc.) were used for in vivo tests. The xenograft models of the gastric cancer experiments suggested that silencing of ERBB4 can markedly suppress the proliferation of gastric cancer cells in vivo and prolong the survival time of tumor-bearing nude mice.
In summary, ERBB4 promoted the proliferation of gastric cancer cells via the PI3K/Akt/mTOR/p70S6K signaling pathway. ERBB4 was significantly correlated with poor survival time. Thus, our data revealed that ERBB4 is a potential prognostic factor and a therapeutic target for gastric cancer.

\section{Acknowledgements}

Not applicable.

\section{Funding}

The research was funded by the Medicine and Health Research Foundation of Zhejiang Province (project no. 2017KY018).

\section{Availability of data and materials}

The datasets used during the present study are available from the corresponding author upon reasonable request. 


\section{Authors' contributions}

JX and LG conceived and designed the study. JX, ZQ, LG, GS and JL performed the experiments. JX wrote the paper. GS and $\mathrm{JL}$ reviewed and edited the manuscript. All authors read and approved the manuscript and agree to be accountable for all aspects of the research in ensuring that the accuracy or integrity of any part of the work are appropriately investigated and resolved.

\section{Ethics approval and consent to participate}

All experimental protocols were approved by Institutional Ethics Committee of Zhejiang Provincial People's Hospital (Hangzhou, China). All patients provided a signed informed consent.

\section{Consent for publication}

Not applicable.

\section{Competing interests}

The authors declare that they have no competing interests.

\section{References}

1. Chen W, Zheng R, Baade PD, Zhang S, Zeng H, Bray F, Jemal A, $\mathrm{Yu}$ XQ and He J: Cancer statistics in China, 2015. CA Cancer J Clin 66: 115-132, 2016.

2. Ferlay J, Shin HR, Bray F, Forman D, Mathers C and Parkin DM: Estimates of worldwide burden of cancer in 2008: GLOBOCAN 2008. Int J Cancer 127: 2893-2917, 2010.

3. Siegel R, Naishadham D and Jemal A: Cancer statistics, 2013. CA Cancer J Clin 63: 11-30, 2013.

4. Ni CY,Murphy MP, Golde TE and Carpenter G: gamma-Secretase cleavage and nuclear localization of ErbB-4 receptor tyrosine kinase. Science 294: 2179-2181, 2001.

5. Sordella R, Bell DW, Haber DA and Settleman J: Gefitinib-sensitizing EGFR mutations in lung cancer activate anti-apoptotic pathways. Science 305: 1163-1167, 2004.

6. Tan WL, Jain A, Takano A, Newell EW, Iyer NG, Lim WT, Tan EH, Zhai W, Hillmer AM, Tam WL, et al: Novel therapeutic targets on the horizon for lung cancer. Lancet Oncol 17: e347-e362, 2016

7. Li M, Zhang Z, Li X, Ye J, Wu X, Tan Z, Liu C, Shen B, Wang XA, $\mathrm{Wu} \mathrm{W}$, et al: Whole-exome and targeted gene sequencing of gallbladder carcinoma identifies recurrent mutations in the ErbB pathway. Nat Genet 46: 872-876, 2014.

8. Arteaga CL and Engelman JA: ERBB receptors: From oncogene discovery to basic science to mechanism-based cancer therapeutics. Cancer Cell 25: 282-303, 2014

9. Vlacich G and Coffey RJ: Resistance to EGFR-targeted therapy: A family affair. Cancer Cell 20: 423-425, 2011

10. Hegde GV, de la Cruz CC, Chiu C, Alag N, Schaefer G, Crocker L, Ross S, Goldenberg D, Merchant M, Tien J, et al: Blocking NRG1 and other ligand-mediated Her4 signaling enhances the magnitude and duration of the chemotherapeutic response of non-small cell lung cancer. Sci Transl Med 5: 171ra18, 2013.

11. Settleman J: A therapeutic opportunity in melanoma: ErbB4 makes a mark on skin. Cancer Cell 16: 278-279, 2009.

12. Ma X, Li L, Tian T, Liu H, Li Q and Gao Q: Study of lung cancer regulatory network that involves erbB4 and tumor marker gene. Saudi J Biol Sci 24: 649-657, 2017.

13. Broughton MN, Westgaard A, Paus E, Øijordsbakken M, Henanger KJ, Naume B and Bjøro T: Specific antibodies and sensitive immunoassays for the human epidermal growth factor receptors (HER2, HER3, and HER4). Tumour Biol 39: 1010428317707436, 2017.

14. Song G, Zhang H, Chen C, Gong L, Chen B, Zhao S, Shi J, Xu J and Ye Z: miR-551b regulates epithelial-mesenchymal transition and metastasis of gastric cancer by inhibiting ERBB4 expression. Oncotarget 8: 45725-45735, 2017.
15. Zhang M, Zhang L, Cui M, Ye W, Zhang P, Zhou S and Wang J: miR-302b inhibits cancer-related inflammation by targeting ERBB4, IRF2 and CXCR4 in esophageal cancer. Oncotarget 8: 49053-49063, 2017.

16. Ohashi Y, Kumagai K, Miyata Y, Matsubara R, Kitaura K, Suzuki S, Hamada Y and Suzuki R: Overexpression of ErbB4 is an independent marker for lymph node metastasis in Japanese patients with oral squamous cell carcinoma. Oral Surg Oral Med Oral Pathol Oral Radiol 122: 313-321, 2016.

17. Ueda Y, Fujishima H, Hirashita T, Shiroshita H, Etoh T, Inomata $\mathrm{M}$ and Shiraishi $\mathrm{N}$ : Clinical impact of small advanced gastric cancer $(\leq 40 \mathrm{~mm})$ in elderly patients: A retrospective cohort study. Int J Surg 45: 131-137, 2017.

18. Pereira MA, Ramos MFKP, Dias AR, Faraj SF, Yagi OK, Safatle-Ribeiro AV, Maluf-Filho F, Zilberstein B, Cecconello I, de Mello ES, et al: Risk factors for lymph node metastasis in western early gastric cancer after optimal surgical treatment. J Gastrointest Surg 22: 23-31, 2018.

19. Chang JY, Shim KN, Tae CH, Lee KE, Lee J, Lee KH, Moon CM, Kim SE, Jung HK, Jung SA, et al: Comparison of clinical outcomes after endoscopic submucosal dissection and surgery in the treatment of early gastric cancer: A single-institute study. Medicine (Baltimore) 96: e7210, 2017.

20. Bang CS, Park JM, Baik GH, Park JJ, Joo MK, Jang JY, Jeon SW, Choi SC, Sung JK and Cho KB: Therapeutic outcomes of endoscopic resection of early gastric cancer with undifferentiated-type histology: A Korean ESD Registry Database analysis. Clin Endosc 50: 569-577, 2017.

21. Pinto C, Di Fabio F, Barone C, Siena S, Falcone A, Cascinu S, Rojas Llimpe FL, Stella G, Schinzari G, Artale S, et al: Phase II study of cetuximab in combination with cisplatin and docetaxel in patients with untreated advanced gastric or gastro-oesophageal junction adenocarcinoma (DOCETUX study). Br J Cancer 101: $1261-1268,2009$.

22. Waddell T, Chau I, Cunningham D, Gonzalez D, Okines AF, Okines C, Wotherspoon A, Saffery C, Middleton G, Wadsley J, et al: Epirubicin, oxaliplatin, and capecitabine with or without panitumumab for patients with previously untreated advanced oesophagogastric cancer (REAL3): A randomised, open-label phase 3 trial. Lancet Oncol 14: 481-489, 2013.

23. Sun GP, Sun Y, Xu RH, Xu JM, Li J, Wang JW, Qin S, Feng JF, $\mathrm{Ba} Y$, Shen L, et al: The Chinese subgroup from a randomized phase III study of lapatinib in combination with weekly paclitaxel versus weekly paclitaxel alone as second-line treatment of HER 2-amplified advanced gastric cancer (AGC) in Asian countries. J Clin Oncol 31 (Suppl): a4109, 2013.

24. Satoh T, Xu RH, Chung HC, Sun GP, Doi T, Xu JM, Tsuji A, Omuro Y, Li J, Wang JW, et al: Lapatinib plus paclitaxel versus paclitaxel alone in the second-line treatment of HER2-amplified advanced gastric cancer in Asian populations: TyTAN - a randomized, phase III study. J Clin Oncol 32: 2039-2049, 2014.

25. Lieto E, Ferraraccio F, Orditura M, Castellano P, Mura AL, Pinto M, Zamboli A, De Vita F and Galizia G: Expression of vascular endothelial growth factor (VEGF) and epidermal growth factor receptor (EGFR) is an independent prognostic indicator of worse outcome in gastric cancer patients. Ann Surg Oncol 15: 69-79, 2008.

26. Chua TC and Merrett ND: Clinicopathologic factors associated with HER2-positive gastric cancer and its impact on survival outcomes - a systematic review. Int J Cancer 130: 2845-2856, 2012.

27. Hayashi M, Inokuchi M, Takagi Y, Yamada H, Kojima K, Kumagai J, Kawano T and Sugihara K: High expression of HER3 is associated with a decreased survival in gastric cancer. Clin Cancer Res 14: 7843-7849, 2008

28. Liu X, Zhou X, Xu H, He Z, Shi X and Wu S: SLC34A2 regulates the proliferation, migration, and invasion of human osteosarcoma cells through PTEN/PI3K/AKT signaling. DNA Cell Biol 36: 775-780; Epub ahead of print, 2017.

29. Liang L, Gao C, Li Y, Sun M, Xu J, Li H, Jia L and Zhao Y: miR-125a-3p/FUT5-FUT6 axis mediates colorectal cancer cell proliferation, migration, invasion and pathological angiogenesis via PI3K-Akt pathway. Cell Death Dis 8: e2968, 2017.

30. Fruman DA, Snapper SB, Yballe CM, Davidson L, Yu JY, Alt FW and Cantley LC: Impaired B cell development and proliferation in absence of phosphoinositide 3-kinase p85alpha. Science 283: 393-397, 1999. 